\title{
BRIEF STATISTICAL ANALYSIS BETWEEN THE ITALIAN NATIONAL PANDEMIC TREND AND THE EFFECTS OF VACCINAL PROPHYLAXIS
}

\author{
Author: Daniele Cataldi ${ }^{1}$ \\ Doctor in Nursing Sciences - Independent Researcher ${ }^{1}$ \\ daniele77c@hotmail.it ${ }^{1}$
}

DOI: 10.26821/IJSRC.9.8.2021.9823

\begin{abstract}
The importance of vaccines is recognized in the international sphere, and certainly not questionable; Many diseases that were once mortal or able to determine great problems for human health, have been eradicated, thanks to their use. In the new disease called COVID-19, discovered due to the propagation of a new Coronavirus (SARS-COV-2), saw the infection proposed quickly to become pandemic, arriving to determine dead and an imposing number of Infected people. Between 3 March 2020 and 10 June 2021, the Italian National Institute of Nuclear Physics, published a series of statistical results, inherent in the national pandemic trend. These data are extremely interesting, but await a first analysis, to understand what the progress of the infection for SARS-COV-2 in Italy and its relative pandemic aspects, in relation to the vaccination on a national scale. Can you have had vaccinal prophylaxis, a specific influence against the spread of the new Coronavirus? In this study, the pandemic data (new cases of infection, number of deceased patients, number of hospitalized patients, number of curved patients, number of patients admitted to intensive care) and those relating to the first and At the second vaccination dose, the results of which are presented for the first time in this study. A study asking questions and seeks to provide hypotheses.
\end{abstract}

Keywords: SARS-CoV-2, COVID-19, Italy, Pandemic Evolution, Vaccinal Prophylaxis.

\section{PREMISE}

Coronavirus (VOC) are a wide family of respiratory viruses that can cause mild to moderate diseases, from Daniele Cataldi, Volume 9 Issue 8, pp 66-74 August 2021 the common cold to respiratory syndromes such as Mers (Middle East Respiratory Syndrome, Middle East Respiratory Syndrome) and SARS (severe acute respiratory syndrome, Severe acute respiratory syndrome).

They are called so for crown-shaped tips that are present on their surface [13].

Human coronavirus known to today, common around the world, are seven, some identified several years ago (the first half of the sixties) and some identified in the new millennium.

\section{Common human coronavirus:}

- 229E (coronavirus alpha).

- NL63 (coronavirus alpha).

- OC43 (coronavirus beta).

- HKU1 (coronavirus beta).

Other human coronavirus:

- MERS-CoV (The Coronavirus beta that causes the Middle East Respiratory Syndrome).

- SARS-CoV (The Coronavirus beta that causes the Severe Acute Respiratory Syndrome).

- SARS-CoV-2 (The Coronavirus causing the COVID-19) [13].

The Coronavirus 2 from severe acute respiratory syndrome, abbreviated in SARS-COV-2 (severe acute respiratory syndrome coronavirus 2) [2] [3], previously appointed new coronavirus of 2019 (2019-nCoV [5]), is a viral strain of the coronavirus species related to the Sars belonging to the genus betacoronavirus (coronaviridae family), subgenere sarbecovirus, discovered around the end of 2019; This is the seventh 
Volume 9 Issue 8 August 2021

coronavirus recognized capable of infecting human beings [3].

The official name given by the OMS - World Health Organization to the syndrome caused by the virus is COVID-19 (COronaVIrus Disease-2019) [4]. The virus was genuinely sequenced after a nucleic acid test carried out on a sample taken from a patient struck by a pneumonia, whose cause was not known, at the beginning of the pandemic of 2019-2021 in Wuhan, in China $[6][7][8]$.

Towards the end of January 2020 the virus spread to Bangkok (Thailand), Tokyo (Japan), Seoul (South Korea), China, Taiwan, Hong Kong, Macao, Malaysia, Japan, United States, Vietnam, Singapore, France, Germany, Australia, Canada, Nepal, Cambodia, Sri Lanka, Italy, United Arab Emirates, United Kingdom, Brazil, Russia and Spain [9].

Although the virus transmission methods were not yet clear, it was confirmed that it is able to go from person to person.

A public health official in the state of Washington in the United States noted that coronavirus are broadcast mainly "through a close contact with another individual, in particular to be treated and sneezing on someone else who is within a radius of about 1-2 meters from that person" [10].

Although respiratory viruses are usually transmitted when the sick subject also has symptoms, it would seem that the SARS-COV-2 can spread even during a close contact with an asymptomatic patient [11].

It is estimated that the basic reproduction number of virus tra

nsmission from person to person is between 1.4 and 3.8. This value indicates the number of other people to which a newly infected patient can transmit the disease.

According to reportedly, the new Coronavirus was so far able to transmit to a chain up to a maximum of four people [12].

As is obvious, therefore the transmission of the virus takes place mainly by air, or by contact with our mucous membranes.

It is therefore a matter of limiting this possibility of contact and entry into our body, using Individual Protection Devices (IPD), as well as the use of lifestyles adapted to this problematic (washing and disinfection of the hands, frequent disinfection of the surfaces In contact with the persons on which the virus can settle, use of specific antiseptics in living, work or hospital environments, etc.), adopting behaviors that can go to limit the possibility of contagion even with social spacing; But the mitigation of the diffusion of this new virus, also depends on other factors, which until today were not considered by health policies at non-Italian but also global level policies.

The transmission of this infection seems to be homogeneous within the European continent, because cases of infection have presented the same trend with respect to the temporal context in which they were observed. In the next chapters these data will be analyzed and we will try to provide hypothetical explanations with respect to such pandemic behavior.

As regards the trend of the Italian pandemic, in addition to having indicated a seasonality [20], with a clear sensitivity to solar radiation, showed microvariations of pandemic increase in the period in which the period in which the First vaccine doses, just like we'll see.

\section{0 - METHOD}

The data relating to the Italian national pandemic eyolution, were extrapolated by the National Institute of Nuclear Physics - INFN Italian [1], between 3 March 2020 and 10 June 2021 [24], and in this study analyzed to understand and understand what was the national pan-demico behavior in relation to vaccination, which began on 27 December 2020, and still in progress.

This study will analyze the data down processed by the INFN, since it is a simple statistical analysis.

\section{1 - Pandemic data analysis}

In relation to the data obtained from the INFN - italian National Institute of Nuclear Physics, the first data are those relating to the "general" trend in the pandemic in Italy, whose data are counted in Fig. 1. From the national trend "General", the data was then divided by the three italian macroscopic areas, or "north (Fig. 2)", "center (Fig. 3)", and "south (Fig. 4)", of the italian Peninsula. 


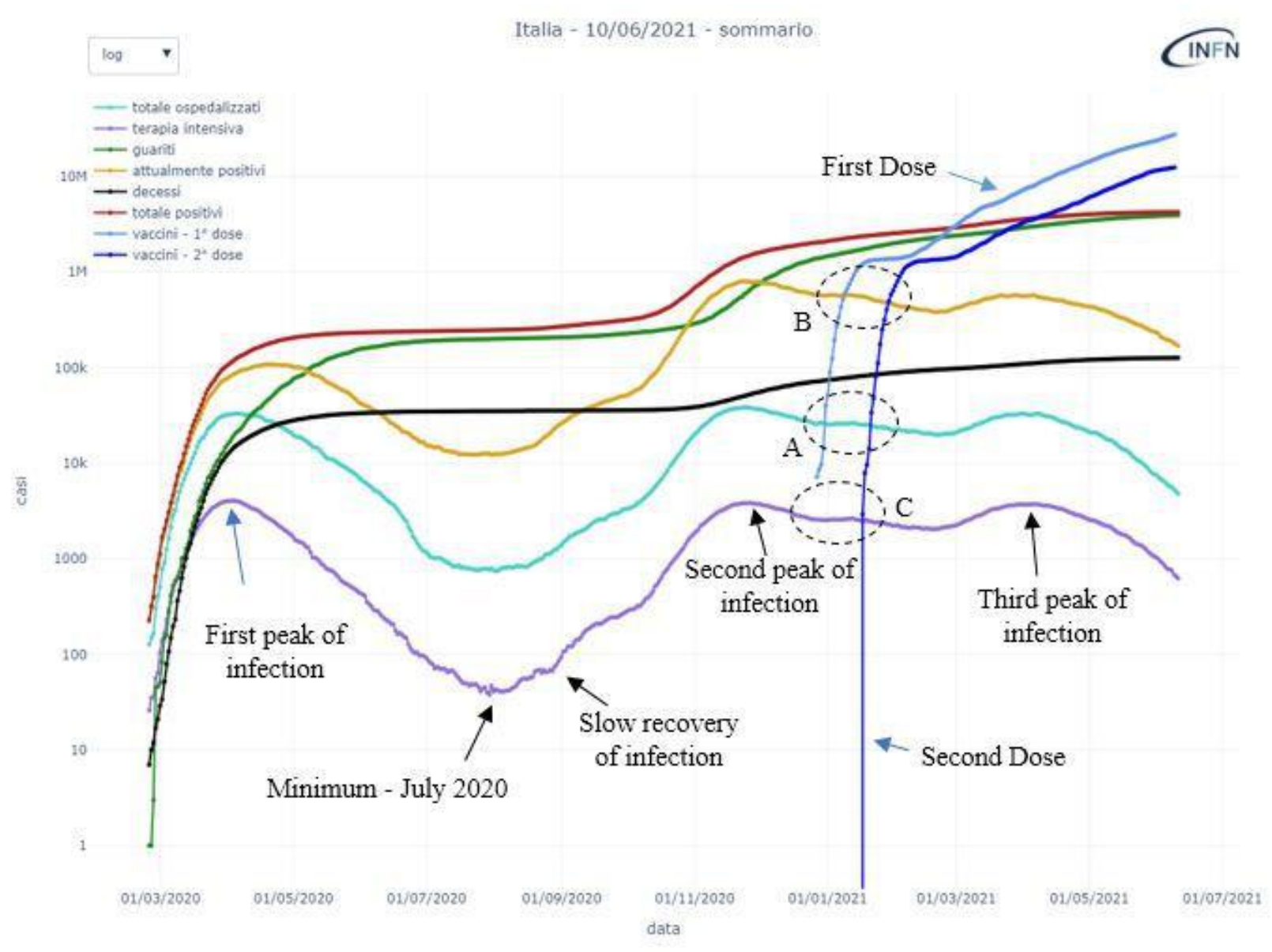

Fig. 1 - "General" Italian pandemic trend, in relation to the cases of identified infection and vaccinations made (first and second dose). On the Cartesian axis of the ordinates we have the number of cases, while on the Cartesian axis of the Ascisses we have the temporal context to which the data refer. Credits: courtesy of the National Institute of Nuclear Physics -Covidstat -https://covid19.infn.it/.

The Fig. 1, It shows how the italian pandemic trend has had several main peaks of the infection to the SARS-COV-2, as well as the re-laundering increases of hospitalized patients, deaths, patients admitted to intensive care and those instead isolated at their domicile.

A trend that is also visible in the three macro main italian areas (North, Central and Southern Italy - Fig. 2, Fig. 3 and Fig. 4).

The first thing that is obvious is that since March 2020, the pandemic then exhausted fasteners to reach the minimum around the end of July 2020 (considering the admissions in intensive care). After that the infection is divided around August 18, 2020, albeit slowly to reach his peak maximum around November 25, 2020.
From this moment on the graph shows an interesting trend. We have the beginning of vaccinations (first dose) on 27 December 2020, and the second dose to start from 17 January 2021. Starting from a few days after the first vaccination dose there is an increase in intensive care shelters (C), an increase in hospitalized patients (A), and an increase in current positives (B).

These increases, although mild tell us in a clear way that the first vaccination was followed in a very tight temporal context re-spent at the first vaccination dose, which there was a slight worsening of the spread of SARS-COV-2 infection also for ef-slice of the the COVID-19 (Fig. 1).

But what determined this increase? According to the temporal context, purely statistical, this increase seems to be side directly with the vaccination action. 


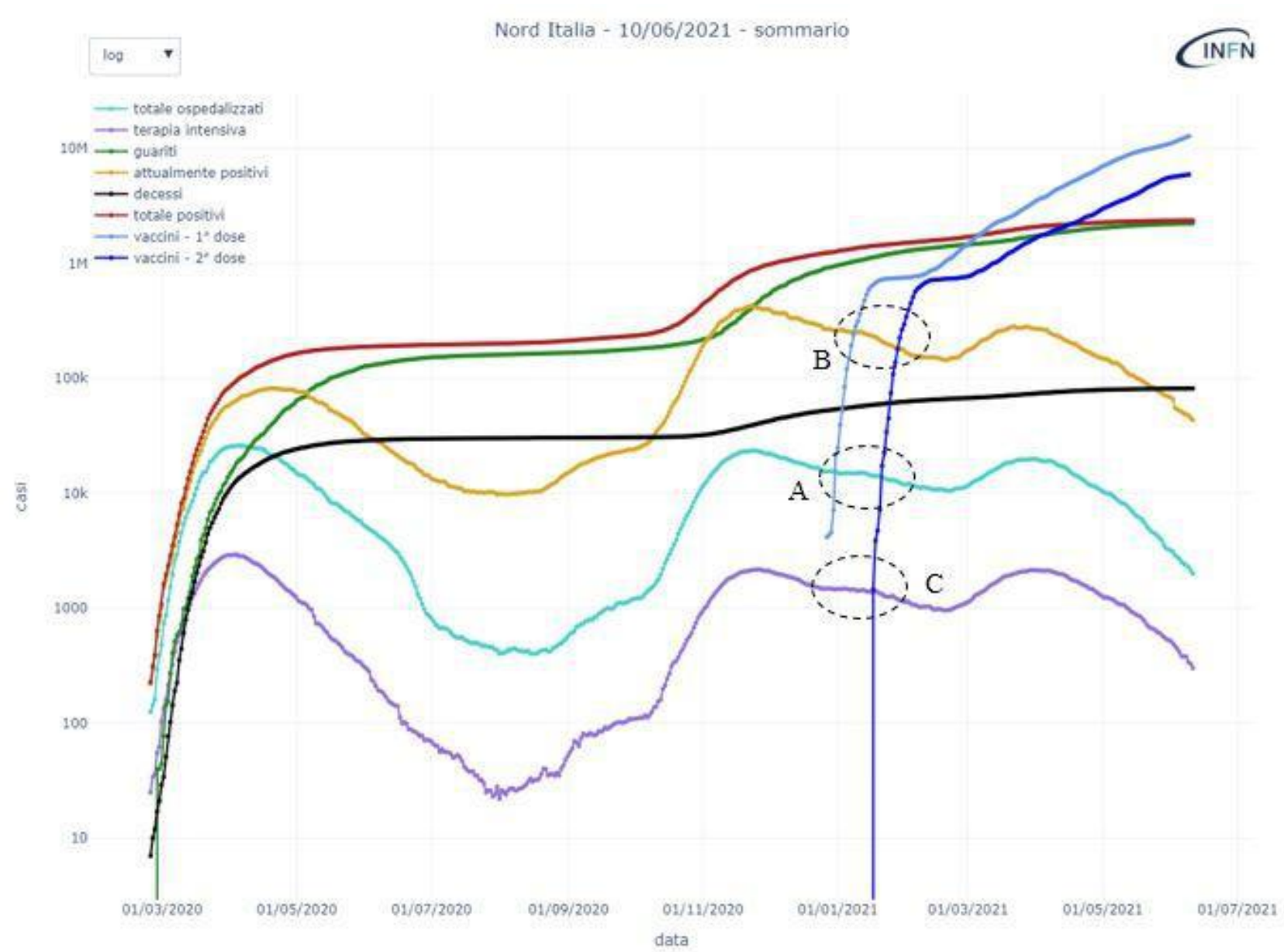

Fig. 2 - Italian pandemic trend "of Northern Italy", in relation to the cases of identified infection and to the effective vaccinations (first and second dose). On the Cartesian axis of the ordinates we have the number of cases, while on the Cartesian axis of the Ascisses we have the temporal context to which the data refer. Credits: courtesy of the National Institute of Nuclear Physics - Covidstat - https://covid19.infn.it/.

This increase, visible in the Fig. 1, 2, 3 and 4, It is override for several weeks up to aveking a third peak of infection and aggravation of the Italian national pandemic, in-back to 6 April 2021.

The data obtained from the National Institute of Nuclear Physics (INFN), are very much Eloquent and explicit.

We would have expected a decrease in the number of contagion after a few days from the second dose, due to the vaccinations with a slight decrease and the contagion, instead of an intense recovery of the spread of the new coronavirus.
On such data it would be advisable to try to provide explanations, which is still science and medicine are not being able to provide.

There may have been a direct effect of the vaccines on the slight increase (A-B-C) observed, while for the third national pandemic peak, the trend could still be mediated by others until today little with sided in Italy, as the seasonality and the very life of the virus itself.

If it were not like this, it would not be explained why the peak is stayed even after the start of vaccinations, to then rush with the beginning of the beautiful season (hot season), just as it happened in 2020, without any prophylaxis vaccinal. 


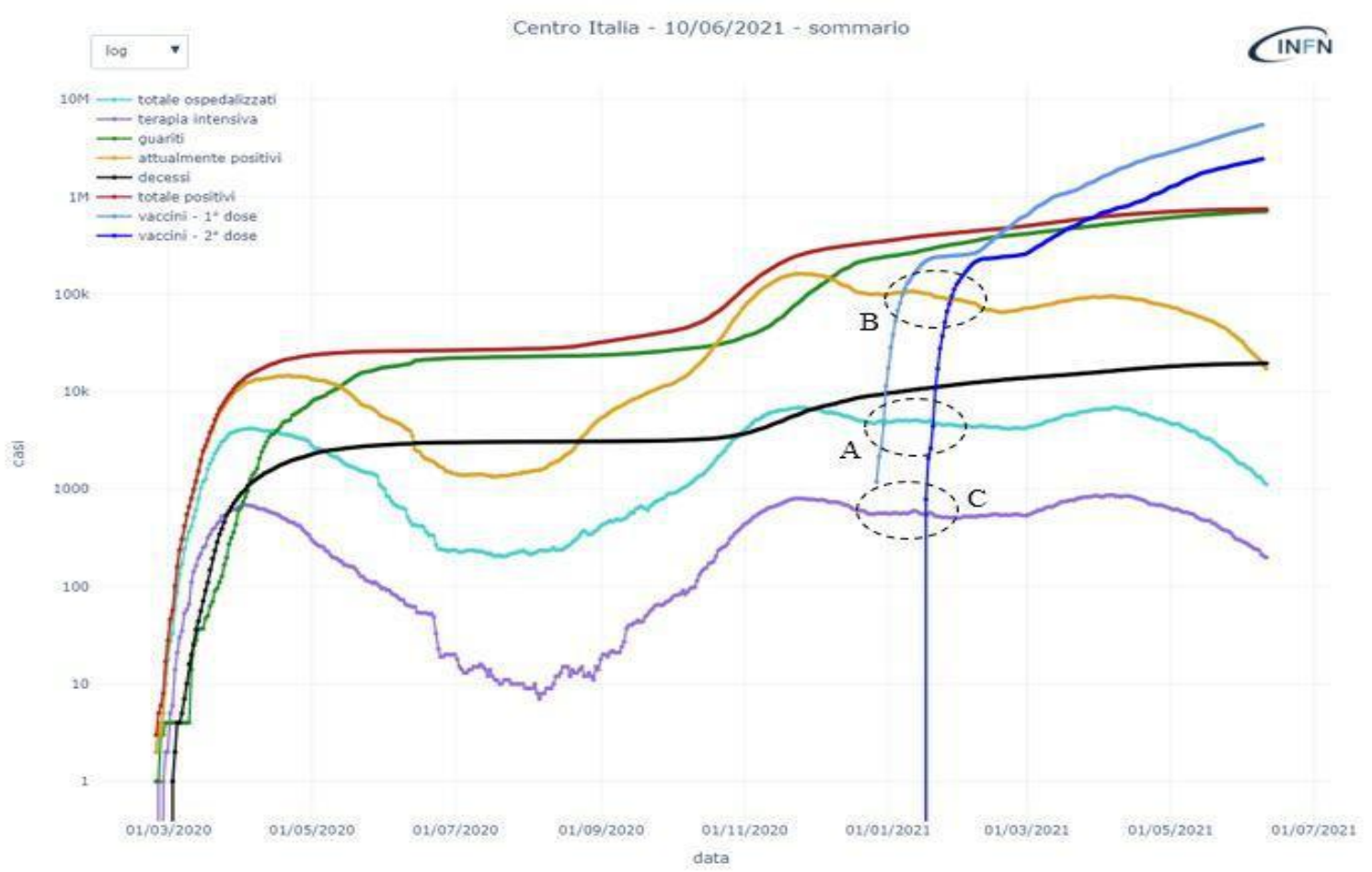

Fig. 3 - Italian pandemic trend "of Central Italy", in relation to the cases of identified infection and to the effettuated vaccinations (first and second dose). On the Cartesian axis of the ordinates we have the number of cases, while on the card axis - they are ascisses we have the temporal context to which the data refer. Credits: courtesy of the National Institute of Nuclear Physics - Covidstat - https://covid19.infn.it/.

The data confirm this correlations even if we observe the pandemic trend of the three macro areas italian separately (Fig. 2, 3 and 4).

The data is very explicit to this sense, it is a clear increase in the infection from SARS-COV-2, compared to the pre-vaccination time period, with a slight ascent of cases of positivity.

This increase indicates that something must have influenced the spread of the virus, if we consider that at that time overlaps the temporal period of the vaccinal lox it is possible to hypothesize the existence of a close relationship between the two phenomena.

\section{2 - Hypothesis}

At this point of the analysis, we can go to consider the specific hypotheses that can hypothetically explain the increases definished by the vaccination action on the Italian pandemic trend. The hypotheses are of different types and can go to consider their own vaccine factors used against infection SARS-COV-2 infection.

$\checkmark \quad$ Unknown and unequaced issues to the patient immune system, which was able to determine an abnormal re-move.

Special effects on the immune system and are also described by pharmaceutical companies [14] and this indicates how the same immune system can react in a non-normal way to vaccination.

$\checkmark \quad$ People with weakened immune system due to some types of cancer treatments and transplant regimes have to face a reduced effectiveness of the vaccine [15] [16].

In this case it is possible that the herself is honored, has had problems with an immune system such as to depauperate so that a possible infection from SARS-COV-2 can then be more easily acquired?

$\checkmark \quad$ There are problems related to example to a poor presence of micronuts that can determine a release of the immune system, but if the patient is not in Knowledge [17].

In this case a similar dysfunction can determine an effective reduction in the body's 
Volume 9 Issue 8 August 2021

defense capacity and therefore make the subject more sensible to any infections, after having taken a vaccination prophylaxis?

$\checkmark$ Other hypotheses concern the effect of the vaccine on our immune system.

Vaccines are normally developed after several years, but in this case the anti-COVID-19 vaccines have been released without a suffocient experimentation [18] whose effects could degrade the immune system and expose it to the most normal viral infections [18] [19].

This could be the reason for which an increase in SARS-COV-2 infections has been observed, after carrying out the first vaccinal dose, exposing the human body to a greater risk of infection? $\checkmark$ The intake of antibiotic drugs concomitant with vaccination may have had effects capable of determining an altered efficacy of the vaccinal profile in developing the immune system to protect from the infection of the SARS-COV-2 [21]?

$\checkmark$ There may be a problem of "immune terference", where vaccinated subjects have developed an immunization on more immunogenic against the SARS-COV-2, due to the effect of vaccinal prophylaxis, determinating in fact a lowering of theirs Immunization capacity [22]?

$\checkmark \quad$ It could be a immunofeficience paradox, post vaccinal, which seems to emerge from some feedback and that would cause a momentary blackout of the immune system to put the patient in an absolute risk condition against all kinds of etiological agent [23]?

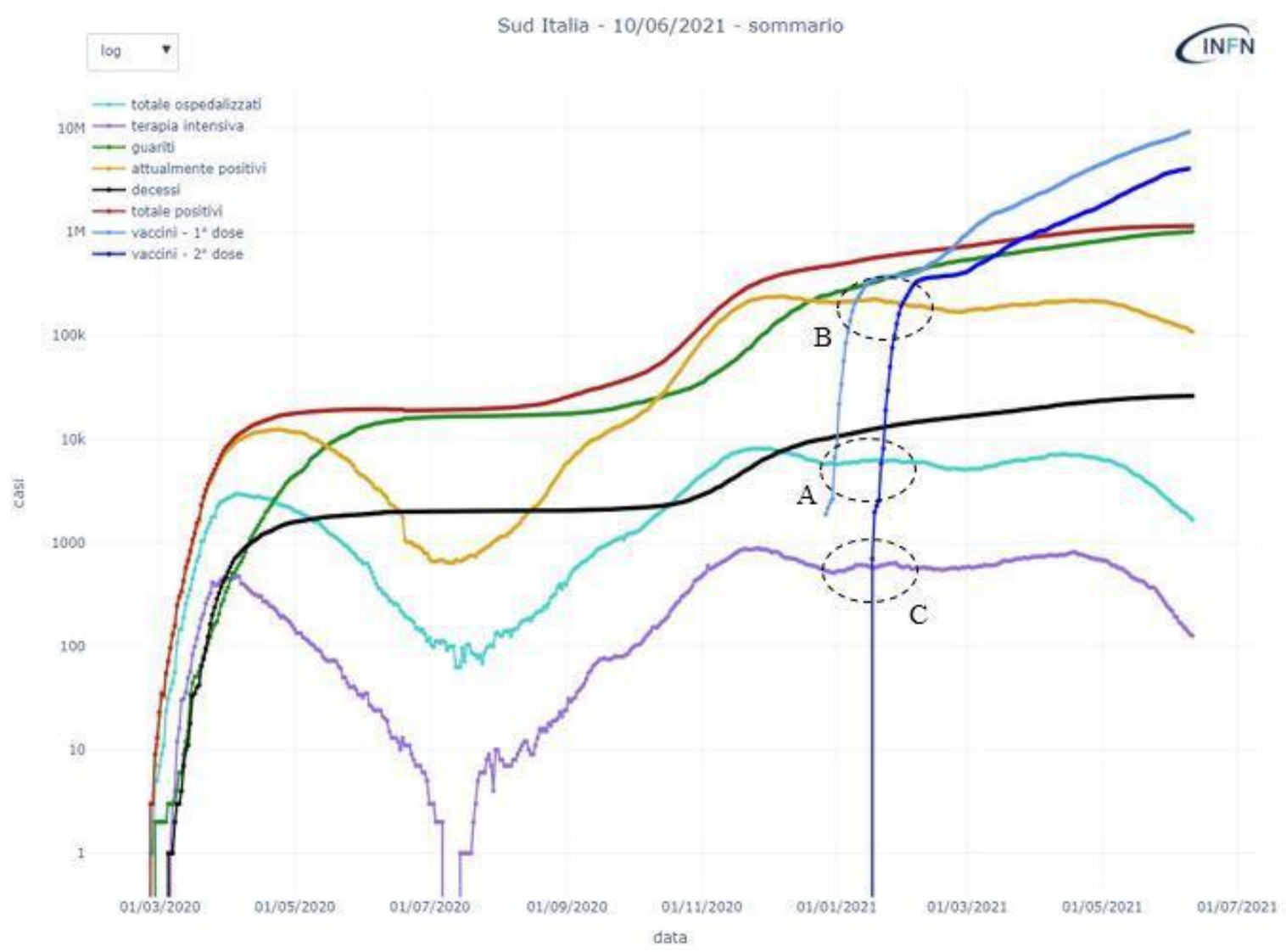

Fig. 4 - Italian pandemic trend "of Southern Italy", in relation to the cases of identified infection and to the effective vaccinations (first and second dose). On the Cartesian axis of the ordinates we have the number of cases, while on the Cartesian axis of the Ascisses we have the temporal context to which the data refer. Credits: courtesy of the National Institute of Nuclear Physics - Covidstat - https://covid19.infn.it/. 


\section{0 - DISCUSSION}

It is now accepted by science, as a healthy immune system, may be able to shield our body from any infection [16] [17].

The data unequivocally demonstrates a slight increase in hospitalizations and cases of infection to the SARSCOV-2 and positivity to the COVID-19, after the specific vaccinal prophylaxis carried out in Italy, between 2020 and 2021.

To this evidence may have influenced meccanisms of the organism, even today unknown or not very considered that we could increase the body's ability to fight this infection decrease? What has this increase determine?

The data indicate a very tight temporal correlation between the start of vaccinations and the increase in cases of infection and hospitalization, in Italy.

\section{0 - CONCLUSIONS}

In conclusion, what are the reasons why, riding between the vaccinal time start of the first and second dose, have been observed in Italy, slight increments of the infection of SARS-COV-2?

On this science and medicine, it should clarify, considering studies more difficult on the effects of vaccinated drugs on the human organism, especially if these have been little experienced in the face of a new little known disease; admitted that these recorded increments (relating to the propagation of SARS-COV2 infection and COVID-19) may have been determined by even little known effects of such drugs or whether it is instead of some other cause. In this context, from a temporal and statistical point of view, the correlation exists.

If you have not dealt with the vaccinal prophylaxis instead to determine these slight increases, which concuses can have resulted in an increase in new coronavirus infections at that time?

Which working hypotheses can be proposed to deepen these facts and try to take and understand more about the progress of the pandemic in Italy?
Or do the data indicate a mere unrelated randomness at all with vaccination data? The role of science is not just to find certain answers, but also to ask questions, sometimes it is not important to be a large number of answers, but ask for sensible questions. This is the role of science and scientific process that in front of questions can attempt to find some answers.

Let's talk about a disease (COVID-19) still little known, of which we know very little, especially in the mechanisms that determine the damage to the human organism. Science does not have to leave any hypotheses, and must deepen these evidence to attempt to mitigate the serious problems that COVID-19 is able to determine the human organism.

In this short study, we tried only to pay attention to an increase in the infection of the SARS-COV-2, on this science and medicine, it should clarify, considering studies more in depth on the effects of vaccinated drugs on the human organism, especially if these have been little experienced in the face of a new little known disease; admitted that these in recorded creations.

Disclaimer: The National Institute of Nuclear Physics, does not assume any kind of responsabilities in relation to the conclusions and deductions of this study.

Thanks: I thank my colleagues, doctors and all health care that in these terrible months have taken care of the patients suffering from COVID-19, curing them and reappropriating them of their lives, a life that within the departments Infectious isolation was full of renunciations and suffering that no one would ever want to live. You must have more respect for the sick, you must have more respect for life, the only thing that really matters, every purpose of ours must face respect for the human person. Only in this way we could achieve a better future, respectful for the individual, for the values and for our human moral. 


\section{REFERENCE}

1. Istituto Nazionale di Fisica Nucleare - INFN https://covid19.infn.it/.

2. Dopo la malattia anche il coronavirus ha un nome, Sars-CoV-2, in ANSA, 12 febbraio 2020.

http://www.ansa.it/canale_scienza_tecnica/not izie/biotech/2020/02/12/dopo-la-malattia-

anche-il-coronavirus-ha-un-nome-sars-cov-2_827d301b-9dfa-441a-a734-

a00e1 aef0777.html.

3. Che cosa è il coronavirus, in Agenzia Giornalistica Italia, 7 marzo 2020. URL consultato il 18 luglio 2020. https://www.agi.it/salute/news/2020-03-

07/coronavirus-cosa-sapere-7372429/.

4. L'OMS ha chiamato COVID-19 la sindrome causata dal nuovo coronavirus, in il Post, 11 febbraio 2020. URL consultato l'11 febbraio 2020 (archiviato l'11 febbraio 2020). https://www.ilpost.it/2020/02/11/covid-19nome-sindrome-nuovo-coronavirus-oms/.

5. Novel Coronavirus (2019-nCoV) Situation Report - 10 (PDF), OMS - Organizzazione Mon-diale della Sanità, 30 gennaio 2020. URL consultato il 18 luglio 2020. https://www.who.int/docs/defaultsource/coronaviruse/situationreports/20200130-sitrep-10-ncov.pdf

6. New-type coronavirus causes pneumonia in Wuhan: expert, su Xinhua, 9 gennaio 2020. URL consultato il 23 gennaio 2020. http://www.xinhuanet.com/english/202001/09/c_138690570.htm.

7. http://www.chinacdc.cn/dfdt/201912/t201912 26_209404.html.

8. https://platform.gisaid.org/epi3/start/CoV202 0 .

9. https://bnonews.com/index.php/2020/01/timel ine-coronavirus-epidemic/.

10. 1Erika Edwards, How does coronavirus spread?

https://www.nbcnews.com/health/healthnews/how-does-new-coronavirus-spread- n1121856.

11. CDC, How 2019-nCoV Spreads, su cdc.gov. URL consultato il $1^{\circ}$ febbraio 2020 https://www.cdc.gov/coronavirus/2019ncov/about/transmission.html.

12. Tina Hesman Saey, How the new coronavirus stacks up against SARS and MERS, su sciencenews.org, 24 gennaio 2020. URL consultato il 25 gennaio 2020 (archiviato dall'url originale il 25 gennaio 2020).

13. L'epidemiologia per la sanità pubblica Istituto Superiore di Sanità https://www.epicentro.-

iss.it/coronavirus/cosa-sono.

14. Allegato I - Riassunto Delle Caratteristiche Del Prodotto - Comirnaty concentrato per dispersione iniettabile - Vaccino a mRNA antiCOVID-19 (modificato a livello dei nucleosidi) - Pfixer/BioNTech.

15. Immunocompromised and Getting the COVID-19 Vaccine - Get vaccinated but discuss ti-ming with your doctor, experts suggest - https:/health.usnews.com/conditions/coronavirus-andyour-health/articles/immunocompromisedand-getting-the-covid-19-vaccine.

16. COVID-19 Vaccines for People with Underlying Medical Conditions - CDC Centers for Disease Control and Prevention. https://www.cdc.gov/coronavirus/2019ncov/vaccines/recommendati ons/-underlying-conditions.-html.

17. Potential roles of micronutrient deficiency and immune system dysfunction in the coronavi-rus disease 2019 (COVID-19) pandemic - Ali Gorji M. D., Maryam Khaleghi Ghadiri M.D.- Nutrition - Volume 82, February 2021, 111047 - DOI: https://doi.org/10.1016/j.nut.2020.111047.

18. Vaccine-and natural infection-induced mechanisms that could modulate vaccine safety - Ronald N. Kostoff, Darja Kanduc, Alan L.Porter, Yehuda Shoen feld, Daniela Calina, Mi-chael B. Briggs, Demetrios A. 
Volume 9 Issue 8 August 2021

Spandidos, Aristidis Tsatsakis - Elsevier Toxicology Reports - Volume 7, 2020, Pages 1448-1458 - DOI: https://doi.org/10.1016/j.toxrep.2020.10.016.

19. Increased Risk of Noninfluenza Respiratory Virus Infections Associated With Receipt of Inactivated Influenza Vaccine - Benjamin J. Cowling, Vicky J. Fang, Hiroshi Nishiura, Kwok-Hung Chan, Sophia Ng, Dennis K. M. Ip, Susan S. Chiu, Gabriel M. Leung, J. S. Ma-lik Peiris Clinical Infectious Diseases, Volume 54, Issue 12, 15 June 2012, Pages 1778-1783, https://doi.org/10.1093/cid/cis307 - Published: 15 March 2012.

20. Mohammad Hossein Nasirpour, Abbas Sharifi, Mohsen Ahmadi \& Saeid Jafarzadeh Ghoushchi - Revealing the relationship between solar activity and COVID-19 and foreca-sting of possible future viruses using multi-step autoregression (MSAR) - Springer - Envi-ronmental Science and Pollution Research (2021) - Published: 16 March 2021. https://link.springer.com/article/10.1007/s11356-021-13249-2.

21. Antibiotics-Driven Gut Microbiome Perturbation Alters Immunity to Vaccines in Humans - Thomas Hagan, Mario Cortese114, Nadine Rouphae, Carolyn Boudreau, Caitlin
Linde, Mo-han S.Maddur, Jishnu Das, Hong Wang, Jenna Guthmiller, Nai-Ying Zheng, Min Huang, Amit A. Uphadhyay, Luiz Gardinassi, Caroline Petitdemange, Michele Paine McCullough, Sara Jo Johnson, Kiran Gill, Barbara Cervasi, Jun Zou, Alexis Bretin, Megan Hahn, Andrew T. Gewirtz, Steve E. Bosinger, Patrick C. Wilson, Shuzhao Li, Galit Alter, Surender Khura-na, Hana Golding, Bali Pulendran. - Cell - Volume 178, Issue 6, 5 September 2019, Pages 13131328.e13.

22. Reduction of Anti-HIV-1 Gag Immune Responses During Co-Immunization: Immune Inter-ference by the HIV-1 Envelope Toapanta, Franklin R.; Craigo, Jodi K; Montelaro, Ronald C; Ross, Ted M. - Current HIV Research, Volume 5, Number 2, 2007, pp. 199-209(11) - Bentham Science Publishers - DOI: https://doi.org/10.2174/157016-207780077057.

23. The paradoxical association between immunodeficiency and autoimmunity: comment on the article by Atkinson - Arthritis \& Rheumatism - Vol. 39, No. 1, January $19 \%$. pp 179-182 - Q 1956, American College of Rheumatology - https://covid19.infn-.it/.

24. Covidstat - https://covid19.infn.it/. 